\title{
Correction to: 3,3'-Diindolylmethane Encapsulated Chitosan Nanoparticles Accelerates Inflammatory Markers, ER/PR, Glycoprotein and Mast Cells Population During Chemical Carcinogen Induced Mammary Cancer in Rats
}

\author{
Isabella Stainsloss $^{1} \cdot$ Mirunalini Sankaran $^{1} \cdot$ Pandiyan Kannaiyan $^{2}$
}

Accepted: 8 May 2021 / Published online: 25 May 2021

(C) Association of Clinical Biochemists of India 2021

Correction to: Ind J Clin Biochem (Oct-Dec 2018) 33(4):397-405 https://doi.org/10.1007/s12291-017-0701-2

The original version of this article published on 23 September 2021, unfortunately contained a mistake. The Fig. 2 panels (d) is incorrectly published.
The correct version of this Fig. 2 panels (d) is given below.

The original article can be found online at https://

doi.org/10.1007/s12291-017-0701-2.

Mirunalini Sankaran

mirunasankar@gmail.com

1 Department of Biochemistry and Biotechnology, Annamalai

University, Annamalai Nagar, Chidambaram,

Tamil Nadu 608 002, India

2 Department of Inorganic Chemistry, University of Madras,

Guindy Campus, Chennai, Tamil Nadu, India 
Fig. 2 a-f shows the effect of DIM and DIM@CS-NP on the histopathological analysis by toludine blue staining respectively. The DMBA induced (b) rats shows showed significantly increased number of mast cell population in the mammary tissues. Here,

DIM@CS-NP 0.5 mg/kg b.wt. d Treatment significantly reduces the number of mast cell population level than DIM mg/ kg b.wt. c However, DIM (e) and DIM@CS-NP (f) alone treated groups of rats no significant changes were absorbed compared to control (a)
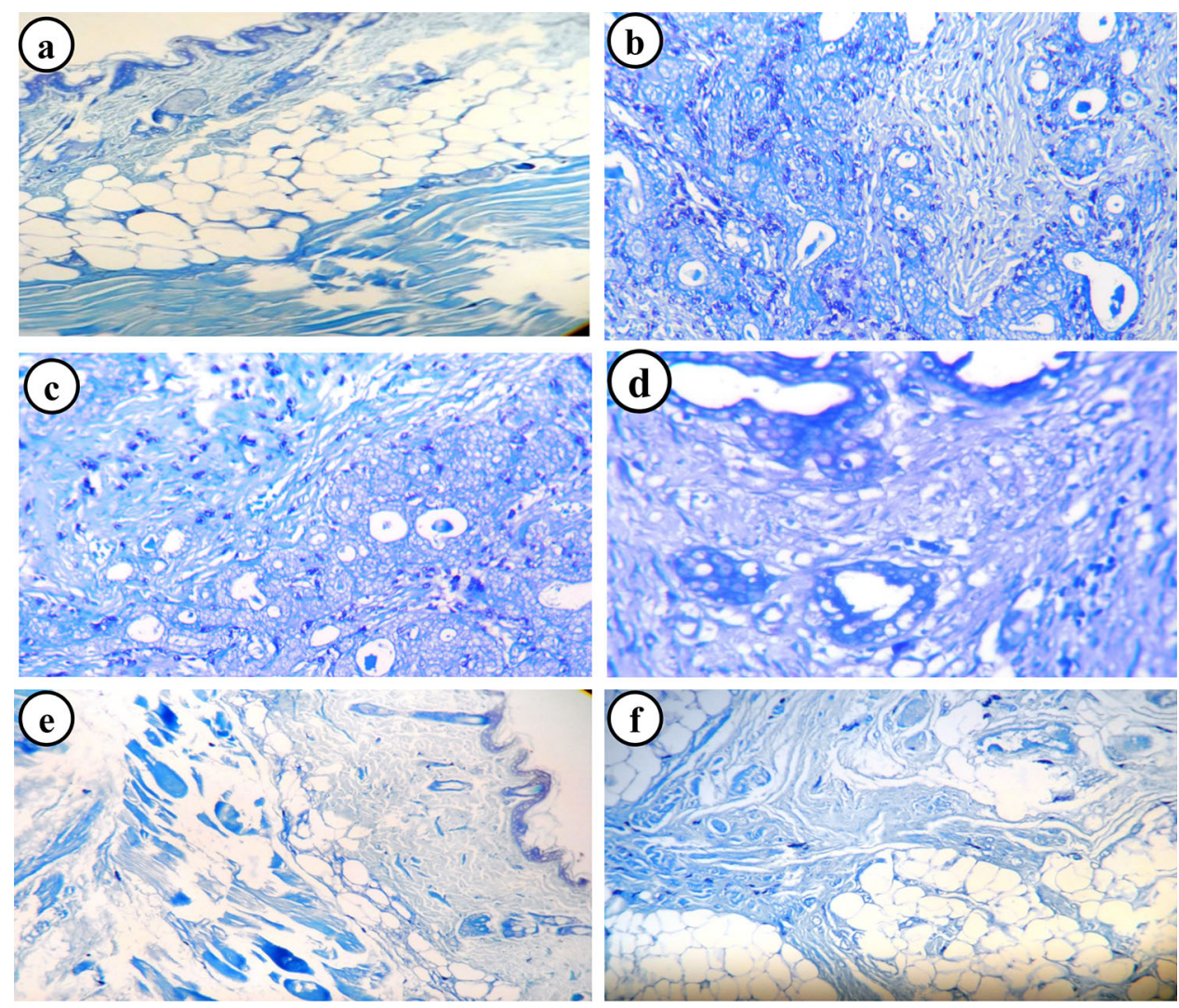